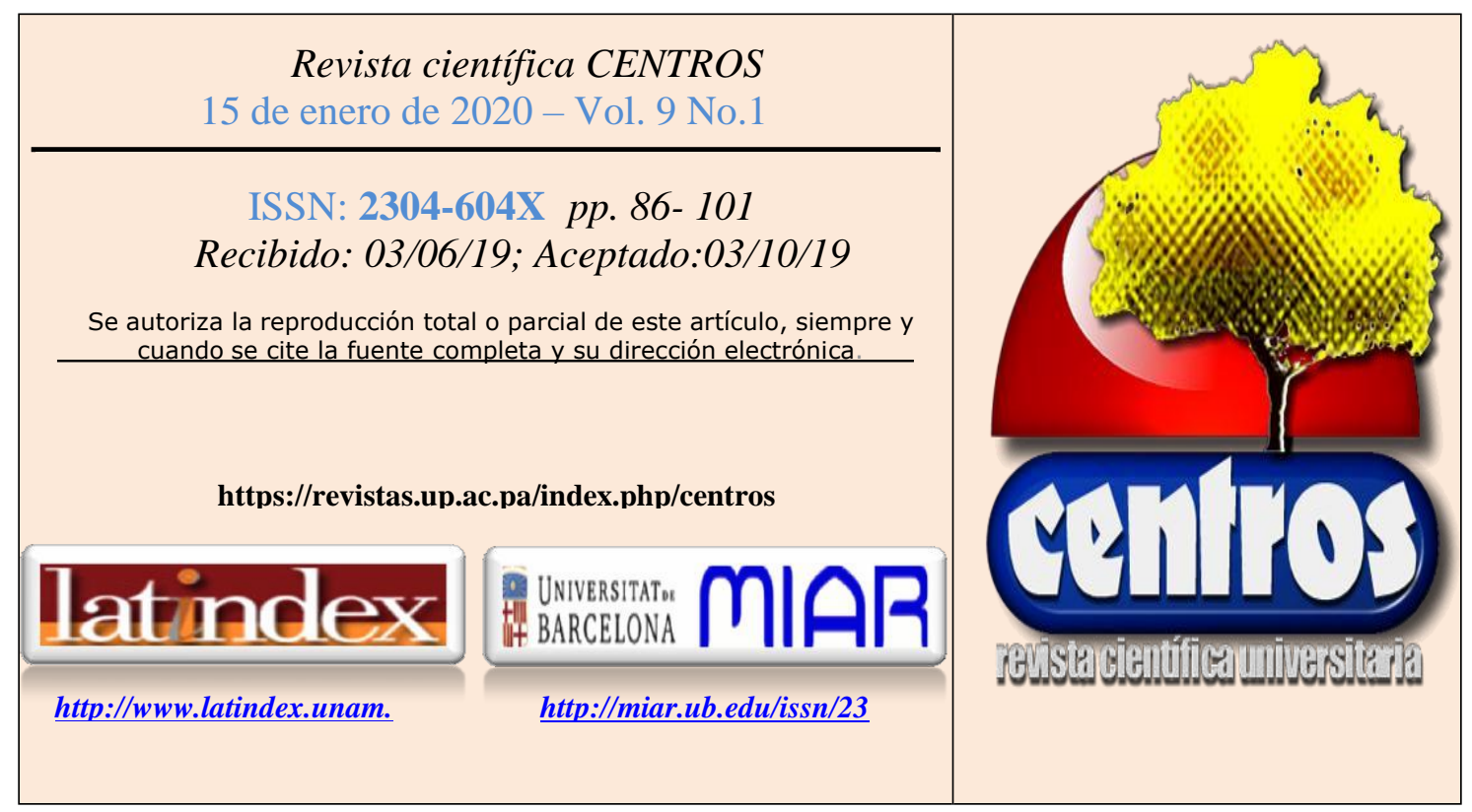

\title{
COMUNICACIÓN SOCIAL DE LAS CIENCIAS: NECESIDAD DE FORMACIÓN EN LA UNIVERSIDAD DE PANAMÁ
}

\section{SOCIAL COMMUNICATION OF SCIENCE: NEED FOR TRAINING AT THE UNIVERSITY OF PANAMA}

Bladimir Enrique Cedeño-Vega ${ }^{1}$, Alcira Prieto-Montero², Yajaira McElfresh³, Nelva Alvarado4, y Jordi Querol-Audí $4,5,6$.

1 Universidad de Panamá, Facultad de Comunicación Social,. Email:

bladimir.cedeno@up.ac.pa; https://orcid.org/0000-0002-4927-0197

2 Universidad de Panamá, Facultad de Comunicación Social, alcira.prieto@up.ac.pa

3 Universidad de Panamá, Facultad de Comunicación Social, Departamento de Comunicación Digital. Email: ymc0712@hotmail.com.

4 Universidad de Panamá, Instituto Especializado de Análisis. Email: nelva.alvarado@up.ac.pa.

5 Universidad de Panamá, Laboratorio de Microbiología Experimental y Aplicada. Email: jordi.querol@up.ac.pa, https://orcid.org/0000-0003-2892-7599.

6 Sistema Nacional de Investigación, SENACYT. 


\title{
RESUMEN
}

El objetivo de este trabajo ha sido analizar y evaluar la necesidad de crear una formación en comunicación de las ciencias en la Universidad de Panamá. Desde esta perspectiva, nos centramos en analizar las posiciones y opiniones de los distintos estamentos involucrados en el proceso de comunicación de las ciencias, dentro y fuera de la institución. Para ello se analizó documentación y resultados de estudios existentes, se realizaron dos entrevistas y se aplicaron tres encuestas a distintos actores del engranaje profesional y académico de la comunicación social y las ciencias y se compararon sus datos. Los resultados obtenidos indican una gran demanda de especialización en comunicación social de las ciencias proveniente tanto de los profesionales que hacen ciencia como de los comunicadores sociales y un gran interés por promover este tema por parte de las instituciones académicas y gubernamentales. Este trabajo justifica la necesidad de crear una formación en comunicación social de las ciencias en la Universidad de Panamá, que forme comunicadores que adquieran capacidades para conocer y dominar las herramientas de la comunicación plural de las ciencias para una mejor difusión de los conocimientos científicos que se producen en Panamá y en el mundo para la ciudadanía panameña.

PALABRAS CLAVE: comunicación científica; formación especializada; Universidad de Panamá.

\begin{abstract}
The objective of this work is to analyze and evaluate the need to create a training in science communication at the University of Panama. From this perspective, we focused on analyzing the positions and opinions of the different estates involved in the process of communication of science, inside and outside the institution. For this, documentation and results from existing studies were analyzed, interviews were conducted and three surveys were applied to different actors of the professional and academic gear of social communication and science and data were compared. Our results indicate a great demand for a specialization in social communication of science from both professionals who do science and social communicators and a great interest in promoting this area by both academic and government institutions.
\end{abstract}


These results justify the need to create a training in social communication of science at the University of Panama in order to form communicators who will acquire the skills and master the tools of plural communication of science to achieve a better dissemination of the scientific knowledge produced in Panama and in the world for Panamanian citizens.

KEYWORDS: Scientific communication, specialized training, University of Panama.

\section{INTRODUCCIÓN}

Según Castellanos (2016), a lo largo de la historia los estudios sobre comunicación han reflejado cómo ciertos procesos de interacción social reclaman explicaciones sobre algunas consideraciones. En este sentido, señala que en las dos últimas décadas, un reclamo ha tomado fuerza en Latinoamérica desde dentro de la actividad pública: "hacer comunicable la ciencia en general con la finalidad de construir conocimiento científico que nos beneficie en todos los ámbitos de nuestra vida." El cual debe ser enmarcado dentro de "un proceso de transcodificación que comprende diversas prácticas comunicativas textuales, visuales y sonoras en ámbitos académicos y fuera de ellos con el propósito de informar, difundir, diseminar o divulgar sobre ciencia" (Quiñonez, 2015).

Sin embargo, este es un tema que no es nada nuevo: desde 1958 en los Estados Unidos la educación científica de la población pasó a ser parte de la agenda de trabajo de las autoridades educativas y de prácticamente todas las asociaciones profesionales, preocupados por el papel que la ciencia y la tecnología debían tener en una nación, tema que se consolidó en 1985, cuando la American Association for the Advancement of Science (AAAS) pone en marcha el proyecto Science for all Americans. Por esa misma época en Europa, el Comité de la Comprensión Pública de la Ciencia (COPUS), que estuvo formado inicialmente por un grupo de investigadores y profesionales vinculados al Science Museum of London, percibió que un público desprovisto de conocimientos e interés por las cuestiones científicas corría el riesgo de convertirse en parte de un movimiento anti-ciencia (Bauer et al, 2007). Visualizándose que, si se quería tener una sociedad científicamente alfabetizada, se debía promover la creación de posgrados y publicaciones académicas especializadas en lo que empezó a denominarse 
como public understanding of science (comprensión pública de la ciencia) (Orozco, 2016). Lo cual, según Bauer, dio lugar al desarrollo de nuevos programas de comprensión y comunicación pública de la ciencia y la tecnología. No obstante, a pesar de los muchos esfuerzos a partir de entonces, los estudios sobre la percepción de la ciencia elaborados en Iberoamérica y Europa en los últimos años (Red de Indicadores de Ciencia y TecnologíaOrganización de Estados Iberoamericanos [RICYT-OEI], 2015), muestran un estancamiento del interés de los ciudadanos en estos temas. Lo que indica, como ya apuntó Castellanos (2008), que todavía queda mucho trecho por recorrer en la relación ciencia-sociedad, lo que refleja que algo se debe estar "haciendo mal en la Comunicación Pública de la Ciencia en todo el mundo" (Castellanos, 2008).

En el caso de Panamá, hasta la fecha se han llevado a cabo cinco encuestas de percepción social de la ciencia y la tecnología, coordinadas por parte de la Secretaría Nacional de Ciencia, Tecnología e Innovación (en adelante SENACYT), aplicadas en los años 2001, 2006, 2008, 2010 y 2017. El análisis de estas cinco encuestas, presentado en el foro "20 años de percepción social de la ciencia en Panamá" de 2018, muestra que la población panameña tiene una fuerte confianza en la ciencia y en la tecnología, así como en los beneficios futuros que promete el desarrollo científico-tecnológico. Que los temas de medicina y salud son aquellos que la enorme mayoría de la población define como de "mucho" o "bastante" interés para mantenerse informada, seguidos por el medio ambiente, la ecología, la ciencia y la tecnología. Sin embargo, a la hora de valorar el nivel informativo de estas disciplinas, las distintas encuestas muestran que la población cree que tiene déficit de información. En este sentido, una gran parte de la población les reprocha a los científicos su falta de compromiso con la comunicación pública de los resultados de sus investigaciones: casi dos tercios está de acuerdo con la idea de que los científicos se esfuerzan poco para informar al público sobre su trabajo, desconsiderando, como dice Lage (2001), que el conocimiento científico es una tarea social. El informe también refleja que un $40 \%$ de la población no está interesada en temas de ciencia y tecnología, siendo las razones personales la mayor causa de ese desinterés. No obstante, la otra razón es que muchas de ellas no entienden los temas de ciencia, lo que podría estar mostrando que existe una dificultad intrínseca por una parte del público de entender los 
materiales divulgativos. Así mismo, la mitad del grupo de población que está interesada en contenidos científicos siente al mismo tiempo que tiene déficit de información sobre estos temas. Esto demuestra que la relación entre la comunidad científica y el público no está siendo tan fluida. Esto supone un desafío para las políticas de comunicación y divulgación científica (SENACYT, 2017).

En cuanto a las estrategias institucionales que intentan acercar las ciencia y la tecnología a la población, encontramos que la SENACYT (2015), dentro de sus programas orgánicos, percibe la ciencia, la investigación, el desarrollo tecnológico y la innovación (CIDTI) como los principales conductores del crecimiento económico y la competitividad, del desarrollo social y de la cultura. En este sentido, lleva a cabo proyectos y programas que están enfocados en potenciar el desarrollo científico y tecnológico del país y de este modo, cerrar la brecha de la desigualdad y fomentar un desarrollo equitativo que mejore la calidad de vida de los panameños. Lo cual lleva consigo un compromiso del gobierno con la ciencia, pero también las obligaciones de la comunidad científica y académica con la sociedad.

Por otro lado, la reciente publicación de la Estrategia de Diplomacia Científica, Tecnológica y de Innovación, por parte de la Cancillería de la República de Panamá (2018 y 2019), que cuenta con el apoyo de la SENACYT, la Autoridad de Innovación Gubernamental, el Instituto de Investigaciones Científicas y Servicios de Alta Tecnología, el Instituto Conmemorativo Gorgas, el Instituto Smithsonian de Investigaciones Tropicales, la Ciudad del Saber y el Programa de las Naciones Unidas para el Medio Ambiente, pone de manifiesto el reconocimiento por parte de las instituciones nacionales de la enorme importancia que las Ciencias, Tecnología e Innovación (CTI) tienen dentro del contexto sociopolítico actual. Todos son conscientes de la necesidad de "promover, generar y difundir conocimientos por medio de la investigación" como propone la United Nations Educational, Scientific and Cultural Organization (UNESCO), (1998). Así mismo, los distintos organismos involucrados en esta temática, concuerdan en que en nuestro país existe la necesidad de vencer la dificultad, por gran parte del mundo académico, de comunicar adecuadamente los resultados a diferentes audiencias. Dado que una correcta comunicación permitiría lograr un acercamiento y difundir la cultura científica a la ciudadanía, así como incentivar la participación de los alumnos, profesores e investigadores en la transferencia de los conocimientos, además de promover el interés por la investigación y desarrollar conocimientos y productos con base sólida. 
En esta misma línea, siendo la universidad pública más grande de la república, con presencia en todas las regiones del territorio nacional, la Universidad de Panamá (2009), ha puesto su empeño en la excelencia y el reconocimiento académico internacional, cuyo propósito es aumentar su competitividad para formar académicos y profesionales útiles a la sociedad, así como organizar y realizar investigaciones que faciliten el desarrollo de las ciencias, la innovación y la tecnología del país hacia el resto del mundo, manteniendo ese papel de impulsora permanente con una labor de continuidad social que corresponde a la universidad (Cuberos, 2004). No obstante, en la institución también se es consciente de la gran necesidad de formar expertos en comunicación científica para desarrollar las plataformas necesarias que permitan un flujo de conocimiento científico entre los diferentes estamentos de la sociedad.

En este sentido, la pregunta que nos planteamos en esta investigación giró en torno a la posibilidad de establecer una formación reglada en comunicación de las ciencias en la Universidad de Panamá. Debido a que se observó que existen diversos elementos que parecen conjugarse favorablemente para abrir un escenario fructífero para el establecimiento de la educación superior de la comunicación de la ciencias, cuya dinámica implica una interrelación entre la actividad pública y los miembros productores, transmisores y comunicadores de las ciencias y, de estos, hacia el resto de la sociedad. De allí el fundamento de este trabajo: hacer un estudio sobre la demanda y necesidades para establecer una formación superior en comunicación de las ciencias en la Universidad de Panamá.

\section{MATERIAL Y MÉTODOS}

Diseño de la encuesta y validación. La encuesta fue elaborada por los expertos en comunicación social coautores de este estudio. Una vez redactada, se validó su calidad en forma y contenido aplicándola a un grupo de 10 estudiantes y docentes de la Universidad de Panamá y se realizaron las modificaciones necesarias en aquellos puntos que presentaban dudas por parte de este grupo representativo.

Universo encuestado. Comprendió tres grupos: (i) participantes del seminario-taller internacional "Investigación científica como herramienta para transmitir conocimientos", 
financiado por la SENACYT y que tuvo lugar en la Universidad de Panamá los días 1 al 5 de Abril de 2019, (ii) docentes de la Facultad de Comunicación Social de la Universidad de Panamá y (iii) miembros de Ciencia en Panamá (CeP) y Asociación Centroamericana de Periodistas Científicos (ACPC).

Recolección de datos. Este trabajo se llevó a cabo en la Ciudad de Panamá en el mes de Abril de 2019. Las encuestas se distribuyeron y recolectaron posteriormente en formato impreso para los dos primeros grupos mencionados anteriormente. Para el último grupo (CeP y ACPC) se dio acceso a la encuesta mediante formulario de Google cuyo enlace se les facilitó mediante WhatsApp.

Análisis estadístico. Una vez recopilados los datos, éstos se transfirieron a una hoja de Numbers (Apple Inc.) en la cual se analizaron y graficaron los resultados correspondientes a cada pregunta realizada en la encuesta.

Entrevistas. Se realizaron entrevistas personales a dos representantes de entidades académicas y gubernamentales: Dr. Eric Santamaría, Director de Postgrado de la Vicerrectoría de Investigación y Postgrado de la Universidad de Panamá, Diciembre de 2018; y Mgstr. María Gabriela Alvarado, Jefa de Información y Relaciones Públicas de la Secretaría Nacional de Ciencia, Tecnología e Innovación, Enero de 2019.

\section{RESULTADOS}

Con el fin de analizar la necesidad de una formación reglada en comunicación científica en Panamá, se confeccionó una encuesta para evaluar el interés de la comunidad académica de la Universidad de Panamá y otras instituciones relacionadas con la ciencia, acerca de la creación de un programa de estudios a nivel de postgrado en este área. Esta se distribuyó a un total de 455 personas, de las cuales contestó el $23.7 \%$. El desglose por grupos es el siguiente: (i) 37 participantes del seminario-taller internacional "Investigación científica como herramienta para transmitir conocimientos", de un total de 50, respondió a la encuesta; (ii) 20 profesores de la Facultad de Comunicación Social respondieron a la encuesta de un total de 70; (iii) de un total de 327 personas que en ese momento conformaban los grupo CeP y ACPC, 51 respondieron a la encuesta. 


\section{Perfil Académico del Universo Encuestado}

En primer lugar, se analizaron los perfiles académicos de los participantes, por grupos y en total. Los resultados se muestran en la Figura 1. Como puede observarse, en general, los perfiles son diversos, con grado de Maestría en su mayoría (33.3\%), seguido por grado de Doctor $(23.2 \%)$, licenciatura (18.5\%) y postgrado (7.4\%). Estos porcentajes se mantienen cuando se analizan los distintos grupos por separado, excepto en el caso de la Facultad de Comunicación Social, donde ninguno de los encuestados que respondió a esta pregunta tiene grado de Doctor y el porcentaje de licenciados es significativamente menor a la media (5\% respecto al $18.5 \%$ general). Sin embargo, hay que resaltar que un $50 \%$ de este grupo (10 encuestados) no proporcionó su grado académico, por lo que los porcentajes no reflejan al grupo en su totalidad.

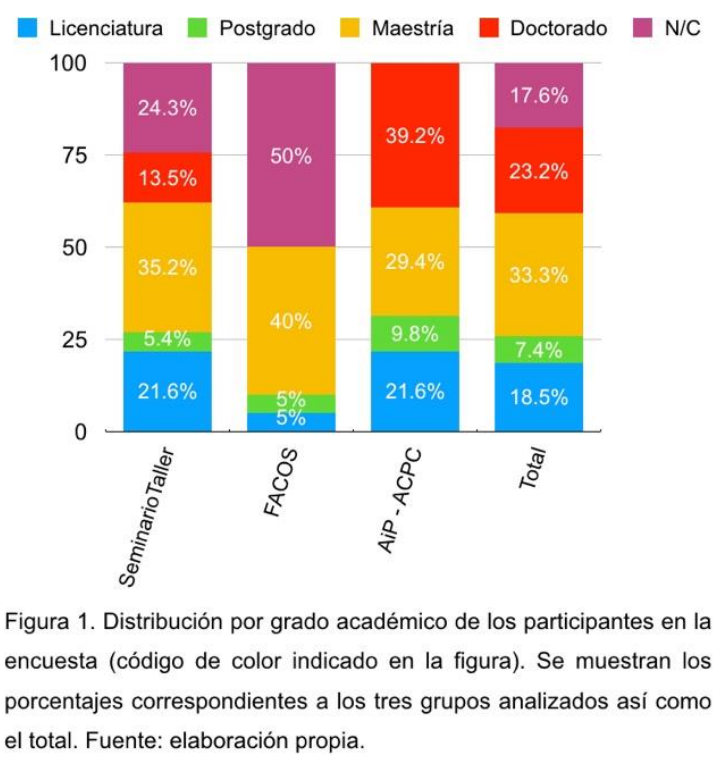

A continuación se muestran los resultados de la encuesta que se aplicó tanto a los miembros de la comunidad académica, los participantes del taller de llustración Científica como a los docentes de la Facultad de Comunicación Social. Esta constó de 5 preguntas clave para evaluar la necesidad de crear un post-grado en comunicación científica, así como el interés de la comunidad académica en que se ofrezca este tipo de formación. 
Nivel de Conocimiento y Participación en Comunicación Científica

Estas dos preguntas evalúan el grado de conocimiento de los encuestados en actividades de comunicación de las ciencias así como su desempeño en este área. Como puede observarse (Figura 2), un gran porcentaje de los encuestados, casi el 60\%, indican que poseen un nivel intermedio, un $10 \%$ declara un nivel alto, y alrededor de un $30 \%$ manifiesta poco o nulo conocimiento. En concordancia con estos resultados, del universo encuestado, el $54.4 \%$ manifiesta haber participado en actividades de comunicación científica (Figura 3).

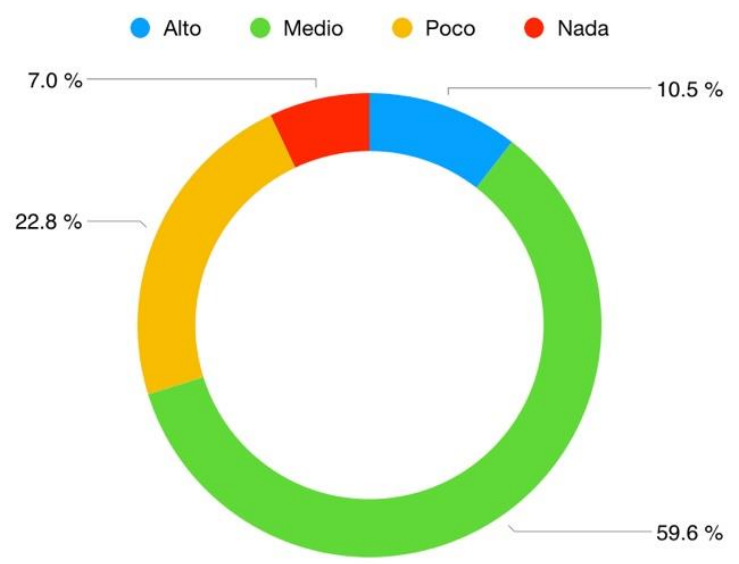

Figura 2. Nivel de conocimiento en comunicación cientifica. Se indica en la figura según código de color con los porcentajes correspondientes. Fuente: elaboración propia.

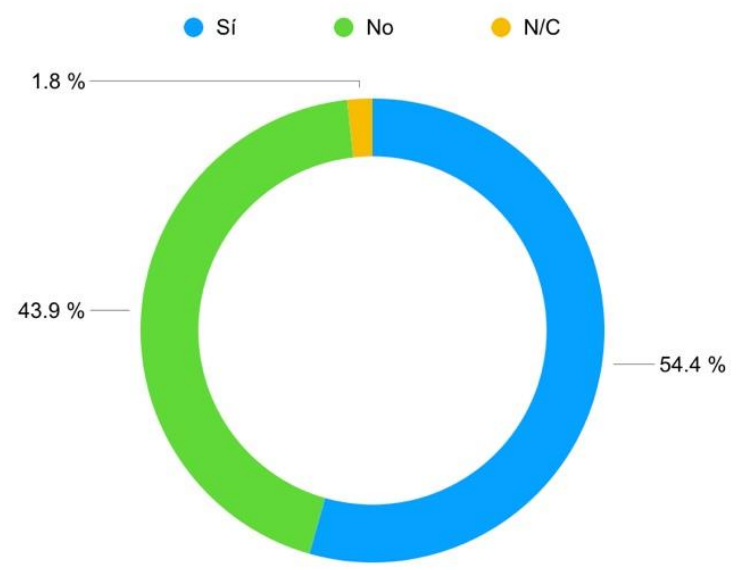

Figura 3. Porcentaje de participación de los encuestados en actividades de comunicación científica (N/C: no sabe/no contesta). Fuente: elaboración propia.

Áreas de Comunicación de La Ciencia 
Los resultados que se muestran en la Figura 4, indican que con aproximadamente un 40\%, la mayoría de los encuestados se enfocan en actividades de enseñanza. El porcentaje se reduce a un $28 \%$ y $24.4 \%$ en las áreas de divulgación y difusión, respectivamente.

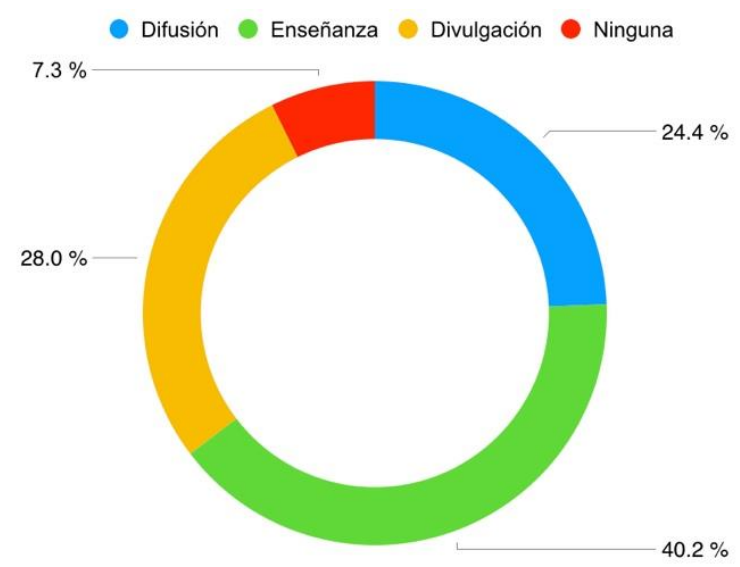

Figura 4. Áreas de comunicación científica en las que ha participado el grupo encuestado. Los porcentajes se muestran en la figura con las áreas correspondientes. Fuente: elaboración propia.

Dificultad para comunicar los resultados. A continuación se les solicitó a los encuestados, evaluar el nivel de dificultad que enfrentaban para transmitir de forma adecuada los resultados científicos a distintas audiencias (Figura 5).

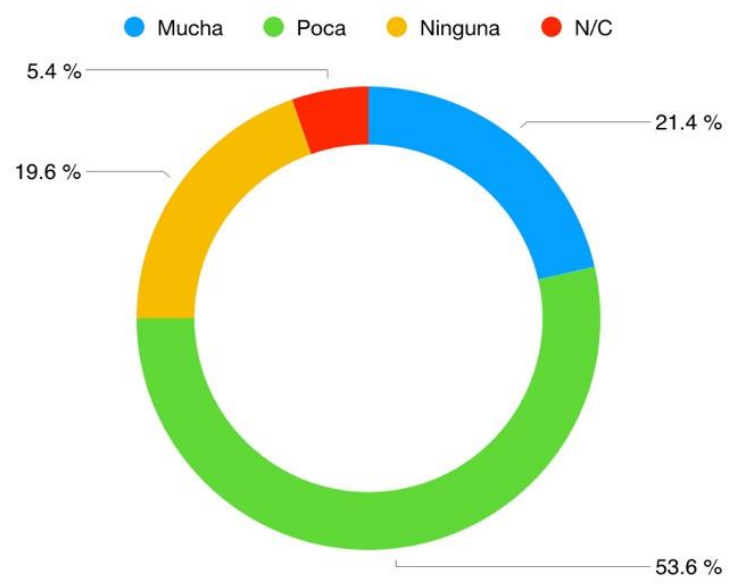

Figura 5. Dificultad para transmitir resultados científicos. Los porcentajes se muestran en la figura (N/C: no sabe/no contesta). Fuente: elaboración propia.

Un 53.6\% declara que les representa poca dificultad, mientras que hay porcentajes similares para aquellos que manifiestan mucha (21.4\%) o ninguna (19.6\%). 
Necesidad de una capacitación formal. A la pregunta de si creen necesaria una capacitación formal en el ámbito de la Comunicación científica en la Universidad de Panamá, el resultado muestra que la gran mayoría del universo encuestado muestra su conformidad con el desarrollo de una oferta académica en este ámbito (Figura 6).

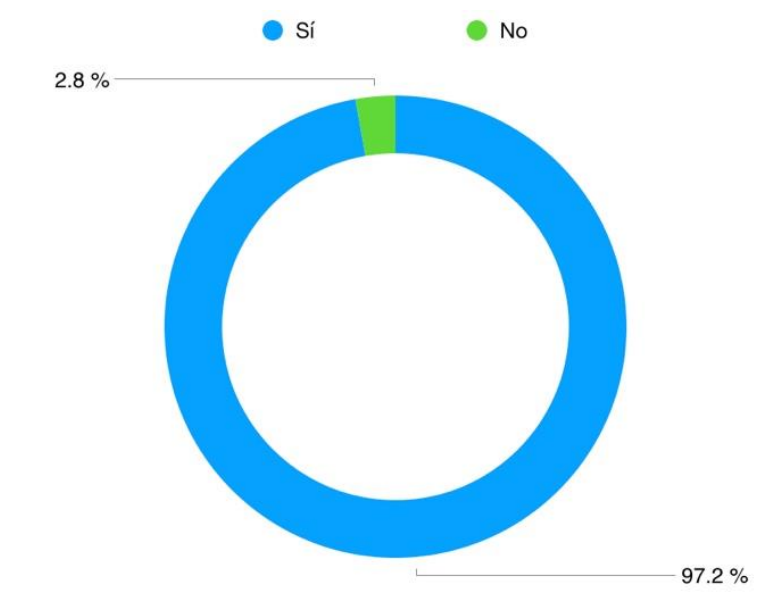

Figura 6. Necesidad de una capacitación formal según el grupo encuestado. Los porcentajes se muestran en la figura (N/C: no sabe/no contesta). Fuente: elaboración propia.

\section{DISCUSIÓN}

Este estudio se centra en evaluar y analizar la necesidad que existe en el país, por parte de los distintos estamentos (gubernamentales, académicos, de investigación) en crear y desarrollar una formación superior reglada que capacite sobre cómo se generan, se gestionan y se transmiten los conocimientos y productos científicos, para hacerlos llegar mejor a los diversos públicos. Después de analizar la documentación y los resultados de los estudios existentes sobre el tema y los datos obtenidos a partir de las entrevistas y las encuestas aplicadas, se observó que en Panamá existe un creciente impulso para obtener conocimientos a través de la actividades de Investigación y Desarrollo. Desafortunadamente, muchos de estos conocimientos no llegan o no se transmiten de manera efectiva a las diversas audiencias, produciéndose así un desaprovechamiento de los esfuerzos invertidos y un distanciamiento entre la ciudadanía, clientes e inversores y el saber científico. En este sentido, a pesar de que la mayoría del universo encuestado tiene experiencia en difusión de los productos de la ciencia en revistas y foros especializados y actividades de carácter académico, existe una preocupación generalizada en el mundo de la ciencia por transmitir adecuadamente los 
conocimientos a otras audiencias. De hacer llegar la ciencia a diversos públicos. De allí que la gran mayoría de los encuestados estén de acuerdo en que es necesario un tipo de formación que otorgue las capacidades para comunicar los conocimientos producto de la ciencia a las distintas audiencias.

Por otro lado, existe una carencia de profesionales especializados en facilitar la comunicación científica a todos los niveles y no solo de la publicación en revistas especializadas; familiarizados con el uso de un lenguaje escrito y visual asequible. Lo que hace propicio fomentar una actividad profesional que estimule el interés por la ciencia y que facilite la transmisión efectiva de los conocimientos adquiridos. Lo que se ha convertido en una de las preocupaciones del mundo de las ciencias en Panamá que busca mecanismos para subsanar esta circunstancia. En este contexto, actualmente no existe en la Universidad de Panamá, ni en el país, una propuesta de formación reglada sobre comunicación científica. Sin embargo, sí existe un gran interés de que se ofrezca una capacitación superior en este ámbito, tanto por las autoridades universitarias, como por grupos multidisciplinares de profesionales que hacen ciencia en el país, y entre docentes de la UP y profesionales de la comunicación social e instituciones públicas.

Por parte de la Dirección de Postgrado de la Vicerrectoría de Investigación y Postgrado de la Universidad de Panamá (Santamaría, 2018) y de la SENACYT (Alvarado, 2019), existe un marcado interés por desarrollar programas de estudios que permitan la preparación académica y profesional en este ámbito de la comunicación. $Y$ entre los diversos grupos que se dedican a la producción de conocimientos y tecnología por medio de la investigación, la transmisión de conocimientos por medio de la docencia y a la comunicación social y el periodismo (CiP, ACPC, Facultad de Ciencias-UP, IEA, FACOS) existe el interés de que se cree una formación superior reglada que capacite sobre cómo se generan, se gestionan y se transmiten estos conocimientos para hacerlos llegar mejor a los diversos públicos; sobre cómo se puede incorporar a la población en general al pensamiento científico para que tengan argumentos válidos para participar en el debate nacional de toma de decisiones y pedir mejores políticas públicas. 
En este sentido, se observó que se ha hecho necesario crear una propuesta de educación superior sólida que pueda ser reconocida por organismos y redes nacionales e internacionales que desarrollen programas de postgrado en el tema. Cuyo objetivo académico sea preparar profesionales capaces de comprender la ciencia y la tecnología como fenómenos complejos y multidimensionales; de generar, gestionar y transmitir el conocimiento científico y las ideas y oportunidades que se derivan de su aplicación y de sus tecnologías asociadas para un mejor aprovechamiento de la sociedad, hacer docencia sobre las formas de transmitir el conocimiento científico, y de participar en el diseño y evaluación de políticas de ciencia y tecnología. Siguiendo esta línea, el Centro Interuniversitario de Desarrollo (1997), recomienda, tomar como referente las propuestas de la Comisión Económica para América latina y el Caribe (CEPAL-UNESCO), ya que el avance educativo debiera articularse no sólo en torno a los objetivos de ciudadanía y competitividad, si no también considerando las políticas de equidad y desempeño.

Desde el punto de vista profesional y académico, en el ámbito de la comunicación y de las ciencias existe la demanda de una formalización especializada de los profesionales con el fin de corresponder a las crecientes necesidades en la comunicación de la producción científica en Panamá. Por un lado, los medios de comunicación generalistas tienen la necesidad de contar con comunicadores con conocimientos en ese campo que sean capaces de transmitir los contenidos de forma eficaz con impacto mediático. Y por otro, en el mundo de la ciencia en Panamá, existe la necesidad de justificar y fortalecer su actividad tanto a nivel nacional como internacional. De allí que se tenga como uno de los principales objetivos popularizar la ciencia mediante la comunicación y la educación, para lo que se necesita entender los diferentes medios y formas de comunicar a diversos públicos. Particularidad que lleva a tener presente lo que dijo James Watson en una entrevista con la BBC: "la comunicación es la esencia de la ciencia" (Paisley, 1984).

En cuanto al desarrollo social, nos encontramos con la necesidad de la popularización de las ciencias, tanto a nivel de población como institucional. En el seno de la ciudadanía nos encontramos con una situación dispar: por un lado, existe un público instruido que demanda una mejor información, accesible pero rigurosa desde el punto de vista científico a los investigadores y a los medios generalistas, a la par que existe una gran masa a la cual esta información no le está llegando, ya sea porque no han sido considerados objeto de esta 
información o porque no están científicamente alfabetizados o porque no se ha logrado vencer la realidad del interés de este público. No obstante, desde el mundo de la ciencia y la comunicación se ha visto la necesidad de que la ciudadanía sea más responsable en la toma de decisiones a nivel social y como nación. Para lo cual es necesario proveerla de conocimientos fiables, de incorporarlos al pensamiento científico para que tengan argumentos válidos para participar en el debate nacional de toma de decisiones y pedir mejores políticas públicas.

\section{CONCLUSIÓN}

En los organismos e instituciones gubernamentales existe la urgente necesidad de alfabetizar a la clase política con respecto a la ciencia para que tomen las decisiones en base a la evidencia y datos científicos. Todo ello hace necesario lograr una mejor difusión de los conocimientos científicos que se producen en Panamá y en el mundo para la ciudadanía. Lo que justifica la necesidad de formar comunicadores de las ciencias que adquieran capacidades para conocer y dominar las herramientas de la comunicación plural, entre otras circunstancias, por la vertiginosa velocidad en que cambia la manera en como el público comparte la divulgación de los conocimientos.

\section{AGRADECIMIENTO}

Los autores agradecen al Dr. Eric Santamaría y la Magíster. María Gabriela Alvarado por concedernos sendas entrevistas.

\section{REFERENCIAS BIBLIOGRÁFICAS}

Alvarado, M. G. (2019). Entrevista. Jefa de Información y Relaciones Públicas de la Secretaría Nacional de Ciencia, Tecnología e Innovación (SENACYT), Panamá, enero 2019.

Bauer, M.; Allum, N. y Miller, S. (2007). "What can we learn from 25 years of PUS survey research? Liberating and expanding the agenda", en Public Understanding of Science, 16(1) 79-95.

Castellanos, P. (2008). Comunicar la ciencia en las sociedades del riesgo. Los medios y los museos de ciencias como mediadores sociales. Razón y Palabra, No. 65. Recuperado de https://bit.ly/2k5jmYy 
Castellanos, V. (2016). Prólogo. Comunicar ciencia en México: Tendencias y narrativas, 201606, pp.7-20. Recuperado de https://www.rei.iteso.mx Centro Interuniversitario de Desarrollo. (1997). Gestión docente universitaria modelos comparados. Recuperado de https://bit.ly/2NGe2Xx

Cuberos-Mejía. R. (2004). Lineamientos para la transformación de una unidad de investigación universitaria. Instituto de Investigaciones de la Facultad de Arquitectura y Diseño de la Universidad del Zulia (IFAD-LUZ), C.I. V-7.608.636. Recuperado de https://bit.ly/32I08sq

Lage Dávila, A. (2001). La ciencia y la cultura: las raíces culturales de la productividad. Educación Médica Superior, 15(2), 189-205. Recuperado de http://scielo.sld.cu/scielo.php?script= sci_arttext\&pid=S0864-21412001000200008\&lng $=$ es\&tlng=es.

Ministerio de Relaciones exteriores. (2018). Cancillería lanza estrategia de diplomacia científica, tecnología e innovación. Recuperado de https://www.mire.gob.pa/index.php/es/noticiasmire/13096-cancilleria-lanza-estrategia-de-diplomacia-cientifica-tecnologica-y-deinnovacion

Ministerio de Relaciones exteriores. (2019). Comunicación científica. Recuperado de https://www.mire.gob.pa/Diplomaciacientifica/

Orozco-Martínez, C. (2016). Tendencias de la investigación académica internacional en la comunicación pública de la ciencia. Comunicar ciencia en México: tendencias y narrativas, 2016-06, pp. 21-52. Recuperado de https://www.rei.iteso.mx

Paisley, W. (1984). Communication in the communication sciences. Dervin y Voigt ed., Norwood, NJ: Progress in communication sciences, vol. V, pp. 1- 43.

Quiñonez, H. (2015). Comunicación Científica: un análisis documental desde la mirada contemporánea. Revista Razón y palabra. Número 90 Junio - agosto, pp. 1-21. Recuperado de https://bit.ly/2O5h86H

Red de Indicadores de Ciencia y Tecnología-Organización de Estados Iberoamericanos. (2015). Manual de Antigua. Red Iberoamericana de indicadores de ciencia y tecnología. Rescatado de https://bit.ly/1TH2D77 
Santamaría, E. (2018). Entrevista. Director de Postgrado de la Vicerrectoría de Investigación y Postgrado de la Universidad de Panamá, diciembre 2018.

Secretaría Nacional de Ciencia, Tecnología e Innovación. (2015). Indicadores de la Política al 2040. Plan Nacional de Ciencia, Tecnología e Innovación.

Secretaría Nacional de Ciencia, Tecnología e Innovación. (10 de julio de 2018). La SENACYT organiza el foro "20 años de percepción social de la ciencia en Panamá. Recuperado de https://bit.ly/2kbaj8H

Secretaría Nacional de Ciencia, Tecnología e Innovación. (2017). V Encuesta de Percepción Social de la Ciencia y la Tecnología 2017.

United Nations Educational, Scientific and Cultural Organization. (1998). Declaración Mundial sobre la Educación Superior en el Siglo XXI: Visión y Acción. Conferencia Mundial sobre la Educación Superior/UNESCO. Recuperado de https://bit.ly/36X1uTn.

Universidad de Panamá. (2009). Modelo educativo y académico de la Universidad de Panamá. Artículo $1^{\circ}$ de la Ley 24 del 14 de junio de 2005. p. 24 